Research Article

\title{
Upregulation of MicroRNA-125b Leads to the Resistance to Inflammatory Injury in Endothelial Progenitor Cells
}

\author{
Ke Yang $(\mathbb{D}$, Xing Liu $(\mathbb{D}$, Wanwen Lin, Yuanyuan Zhang, and Chaoquan Peng $(\mathbb{D}$ \\ Department of Cardiology, The Third Affiliated Hospital, Sun Yat-sen University, Tian-he Road, Guangzhou 510630, China \\ Correspondence should be addressed to Chaoquan Peng; pengcq123456@163.com
}

Received 26 February 2020; Accepted 18 May 2020; Published 14 September 2020

Guest Editor: Shiyue Xu

Copyright ( $) 2020$ Ke Yang et al. This is an open access article distributed under the Creative Commons Attribution License, which permits unrestricted use, distribution, and reproduction in any medium, provided the original work is properly cited.

\begin{abstract}
Objectives. MicroRNA-125b (miR-125b) has been recognized as one of the key regulators of the inflammatory responses in cardiovascular diseases recently. This study sought to dissect the role of miR-125b in modulating the function of endothelial progenitor cells (EPCs) in the inflammatory environment of ischemic hearts. Methods. EPCs were cultured and transfected with miR-125b mimic and negative control mimic. Cell migration and adhesion assays were performed after tumor necrosis factor- $\alpha$ $(\mathrm{TNF}-\alpha)$ treatment to determine EPC function. Cell apoptosis was analyzed by flow cytometry. The activation of the NF- $\kappa \mathrm{B}$ pathway was measured by western blotting. EPC-mediated neovascularization in vivo was studied by using a myocardial infarction model. Results. miR-125b-overexpressed EPCs displayed improved cell migration, adhesion abilities, and reduced cell apoptosis compared with those of the NC group after TNF- $\alpha$ treatment. miR-125b overexpression in EPCs ameliorated TNF$\alpha$-induced activation of the NF- $\kappa$ B pathway. Mice transplanted with miR-125b-overexpressed EPCs showed improved cardiac function recovery and capillary vessel density than the ones transplanted with NC EPCs. Conclusions. miR-125b protects EPCs against TNF- $\alpha$-induced inflammation and cell apoptosis by attenuating the activation of NF- $\kappa \mathrm{B}$ pathway and consequently improves the cardiac function recovery and EPC-mediated neovascularization in the ischemic hearts.
\end{abstract}

\section{Introduction}

The role of EPCs in vascular and tissue repair in ischemic conditions, such as coronary or peripheral vascular diseases, has been well recognized [1]. Circulating EPCs are recruited into the ischemic sites, and they enhance repair through paracrine effects or by incorporating into newly formed vessels after ischemic injury [2-4]. Of note, the functional activities of EPCs are impaired in patients with coronary artery disease (CAD). Compelling evidence suggests that the number and function of EPCs inversely correlate with risk factors for $\mathrm{CAD}$, such as hypertension, diabetes, dyslipidemia, smoking, and age [5-7]. Furthermore, the hostile inflammatory environment in the ischemic sites can induce the apoptosis of EPCs and, consequently, impede the EPCmediated repair $[8,9]$. Hence, improving the function and survival of EPCs in the ischemic sites is critical for EPCmediated repair.
MicroRNAs (miRNAs) are known as a class of noncoding RNAs that modulate the gene expression at a posttranscriptional level. miR-125, known as one of the major regulators in the development of hematological malignancies, is a family of highly conserved miRNAs throughout diverse species [10]. Emerging evidence suggests that miR-125 is involved in the regulation of the innate immune and inflammatory responses $[11,12]$. More importantly, the role of miR-125b in cardiovascular diseases has been drawing increasing attention recently. Dr. Wang et al. reported that the target of miR-125b in the mouse heart is TNF receptor-associated factor 6 (TRAF6), an adaptor molecule in the NF-kB pathway. Overexpression of miR$125 \mathrm{~b}$ in the mouse heart protects the myocardium from ischemia/reperfusion injury by suppressing the TRAF6mediated NF-kB activation [13]. However, the role of miR$125 \mathrm{~b}$ in the regulation of EPCs is still unclear, and further studies are required to study its role. 
In the present study, we focused on the role of miRNA$125 \mathrm{~b}$ in regulating the inflammatory response and function of EPCs in the ischemic hearts. Our results confirmed that upregulation of miRNA-125b ameliorated TNF- $\alpha$-induced functional defects in EPCs in vitro and enhanced EPCmediated neovascularization in the ischemic hearts. miR$125 \mathrm{~b}-\mathrm{mediated}$ inhibition of TNF- $\alpha / \mathrm{NF}-\kappa \mathrm{B}$ pathway activation is involved in the beneficial effects we observed.

\section{Methods and Materials}

2.1. Cell Culture and miRNA Transfection. Bone marrow mononuclear cells were isolated by density gradient centrifugation from the mouse bone marrow and cultured in endothelial cell basal medium-2 (EBM-2) supplemented with endothelial growth medium SingleQuots as indicated by the manufacturer (Clonetics, San Diego). After 4 days of culture, nonadherent cells were removed by washing the plates with phosphate-buffered solution (PBS), and a new medium was applied. EPCs were transfected for $24 \mathrm{~h}$ on day 6 with $50 \mathrm{nmol} / \mathrm{L}$ microRNA mimics for miR-125b (MC10148, Ambion) or miR-negative control (AM17010, Ambion), using the Lipofectamine RNAiMAX reagent (Invitrogen) according to the manufacturer's protocol [13]. Cells were treated with or without $10 \mathrm{ng} / \mathrm{mL}$ tumor necrosis factor- $\alpha$ (TNF- $\alpha$, Peprotech) for $1 \mathrm{~h}$ and then used for the following experiments at day 7. Cultured EPCs were identified by the flow cytometry analysis. Based on the isolation and cultivation protocol, the adherent mononuclear cells were identified as EPCs similar to the previous studies.

2.2. EPC Adhesion to Endothelial Cells In Vitro. $2 \times 10^{5} \mathrm{hu}-$ man umbilical vein endothelial cells (HUVECs) were seeded in each well of a four-well plate $48 \mathrm{~h}$ before the assay to prepare a monolayer of ECs. Then, $1 \times 10^{5}$ EPCs labelled with CM-DiI (CellTrackerTM CM-DiI, Invitrogen) were added to each well and incubated for $3 \mathrm{~h}$ at $37^{\circ} \mathrm{C}$. Nonattached cells were gently washed and removed with PBS, and adherent EPCs were fixed with $4 \%$ paraformaldehyde and counted by independent investigators blinded to treatment randomly.

2.3. EPC Migration In Vitro. A total of $2 \times 10^{4}$ EPCs were harvested and resuspended in $250 \mu \mathrm{L}$ EBM- 2 after TNF- $\alpha$ treatment and pipetted into the upper chamber of a modified Boyden chamber (Costar Transwell assay, $8 \mu \mathrm{m}$ pore size, Corning, NY), which was placed in a 24 -well culture plate containing $500 \mu \mathrm{L}$ EBM-2 medium supplemented with $100 \mathrm{ng} / \mathrm{mL}$ SDF-1. Transmigrated cells were counted after $24 \mathrm{~h}$ incubation at $37^{\circ} \mathrm{C}$ by independent investigators blinded to the treatment randomly.

2.4. EPC Apoptosis Assay. EPCs were treated with $10 \mathrm{ng} / \mathrm{mL}$ tumor necrosis factor- $\alpha$ (TNF- $\alpha$, Peprotech) for $1 \mathrm{~h}$, and cell apoptosis was detected by AnnexinV-staining (Roche, Penzberg, Germany). Briefly, EPCs were cultured with TNF$\alpha(10 \mathrm{ng} / \mathrm{mL})$ for $1 \mathrm{~h}$. Then, EPCs were collected and washed for three times. Annexin V-FITC and propidium iodide (PI) were added to the washed cells $\left(1 \times 10^{6}\right.$ cells $/ \mathrm{mL}$ in FACS buffer) for $15 \mathrm{~min}$ at room temperature in the dark. FACS buffer was added, and cells were analyzed immediately by flow cytometry analysis.

\subsection{Quantitative Real-Time Reverse Transcription Polymerase} Chain Reaction. miR-125b-5p were quantified using specific Taqman assays for miR (Applied Biosystems, USA). Specific primers for miR-125b-5p were obtained from Applied Biosystems. miR-125b levels were normalized to the U6 small nucleolar RNA. Primer sequences for gene encoding for TNF- $\alpha$, IL- $1 \beta$, IL- 6 , and $\beta$-actin were reported in Table 1 . The results were normalized to the mRNA levels of $\beta$-actin.

2.6. Western Blotting. Proteins were extracted with cell lysis buffer (Cell Signaling Technology) and analyzed with by western blotting by using p-NF $\kappa$ B p65 antibody (Ser 276) (1 : 1000, Santa Cruz, sc-101749), NF- $\kappa$ B p65 (1:1000, Cell Signaling, $8242 \mathrm{~T}$ ) and rabbit anti- $\beta$-actin antibodies (1: 3000; Cell Signaling Technology). The intensities of protein bands were quantified densitometrically by using the NIH IMAGE J software.

\subsection{Surgical Induction of Myocardial Infarction (MI) and EPC} Transplantation. The mice were anesthetized by $5.0 \%$ isoflurane, and anaesthesia was maintained by inhalation of $1.5 \%$ to $2 \%$ isoflurane driven by oxygen flow using a rodent ventilator. The hearts were exposed, and the left anterior descending (LAD) coronary artery was ligated with an 8-0 silk ligature. $2 \times 10^{5}$ EPCs suspended in $20 \mu \mathrm{l}$ PBS were injected at 5 different sites at the infarct border zone using an $20 \mu \mathrm{l}$ Hamilton syringe with a 30 -gauge needle. 6-8 mice were used for LAD ligation in each group. The cardiac function was evaluated by echocardiography. The study protocol was approved by the Ethics Committee of Sun Yatsen University.

2.8. Histological Assessments. Cardiac tissues were fixed in $4 \%$ paraformaldehyde for 4 hours and then snapfrozen. Serial cryosectioning was performed starting at $1 \mathrm{~mm}$ below the LAD ligation moving toward the apex. To evaluate PC endothelial differentiation and capillary density, immunohistochemical staining was performed using fluorescent anti-CD31 (Santa Cruz) antibodies. All surgical procedures and pathohistological analyses were performed by investigators blinded to treatment assignments.

2.9. Statistical Analyses. All values are reported as mean\pm SEM. Two-tailed Student's $t$ test was used to compare 2 means. One-way or 2-way ANOVA with a Bonferroni correction was used to compare multiple $(>2)$ means with 1 or 2 independent variables, respectively. $p<0.05$ was considered significant. 
TABle 1: Primer sequences.

\begin{tabular}{lcc}
\hline Gene & Forward primer & Reverse primer \\
\hline TNF-alpha & AGGGATGAGAAGTTCCCAAATG & AGGGATGAGAAGTTCCCAAATG \\
IL-1 $\beta$ & GCAACTGTTCCTGAACTCAACT & ATCTTTTGGGGTCCGTCAACT \\
IL-6 & TCGGAGGCTTAATTACACATGTTC & TGCCATTGCACAACTCTTTTCT \\
\hline
\end{tabular}
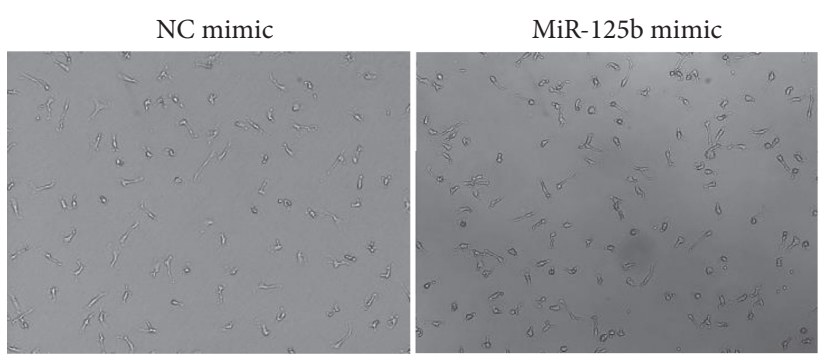

(a)

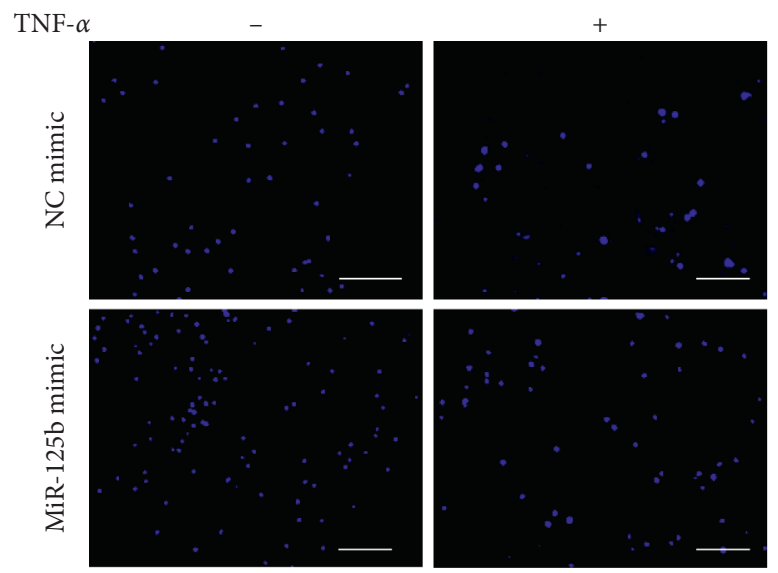

(c)

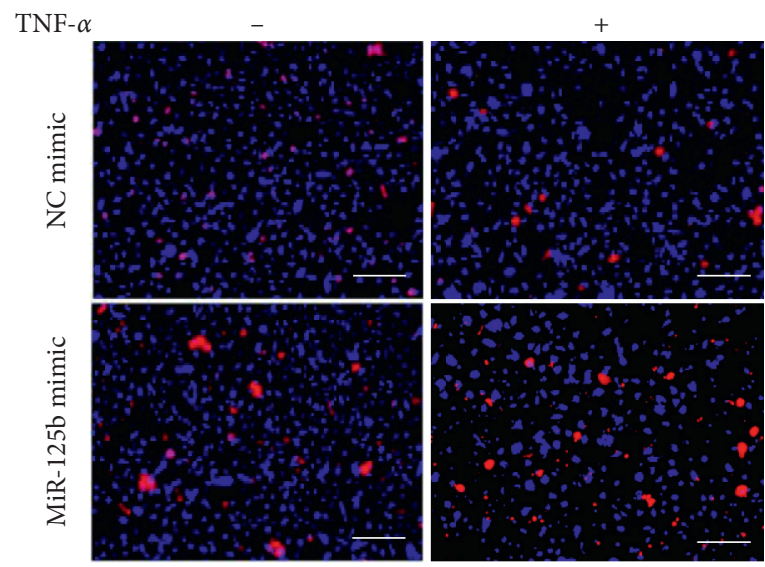

(e)

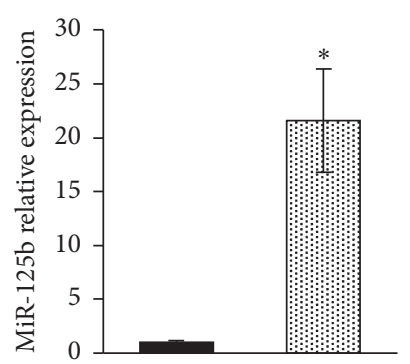

NC mimic

国 MiR-125b mimic

(b)

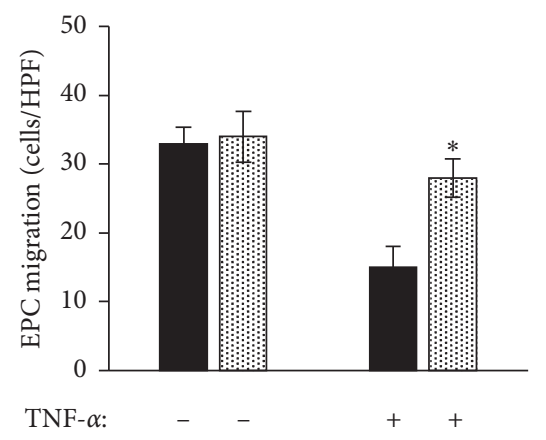

NC mimic

霜 MiR-125b mimic

(d)

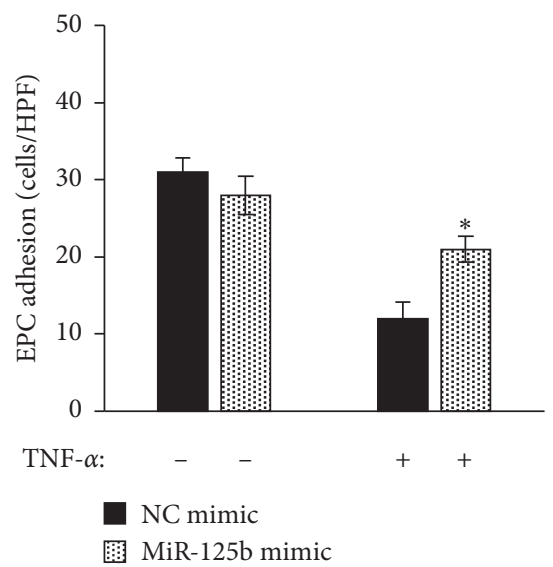

(f)

FIGURE 1: miR-125b overexpression in EPCs preserved its migration and adhesion function after TNF- $\alpha$ treatment. EPCs were transfected with miR-125b mimic and negative control mimic for $24 \mathrm{~h}$. (a) The level of miR125b measured by qRT-PCR (b). EPC migration and adhesion measured after transfection. Representative (c) and quantification (d) of the migratory activity of EPCs. Representative (e) and quantification (f) of DiI-labeled EPC adhesion to HUVECs with TNF- $\alpha$ activation (scale bar $=100 \mu \mathrm{m},{ }^{*} p<0.05$ vs. NC mimic with TNF- $\alpha$ treatment, $n=5)$. 


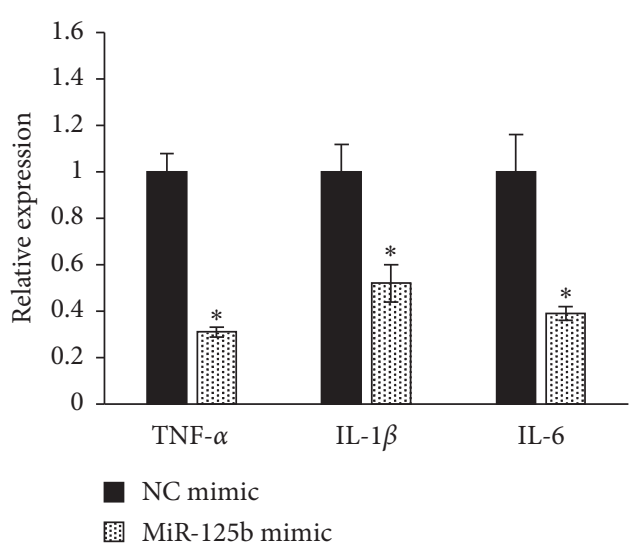

(a)

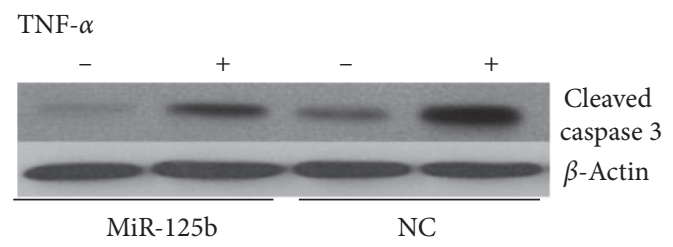

(c)

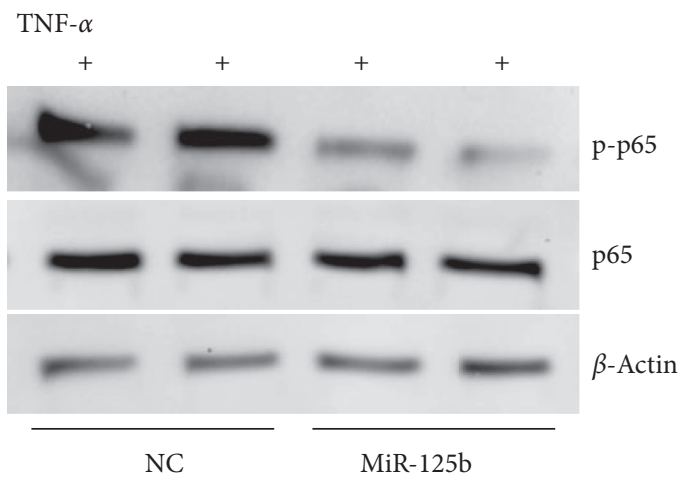

(e)

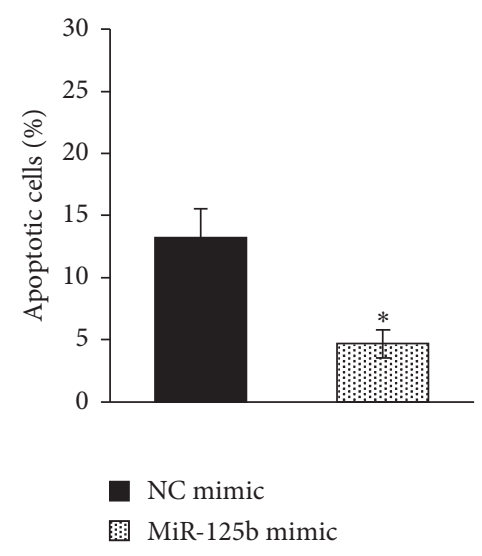

(b)

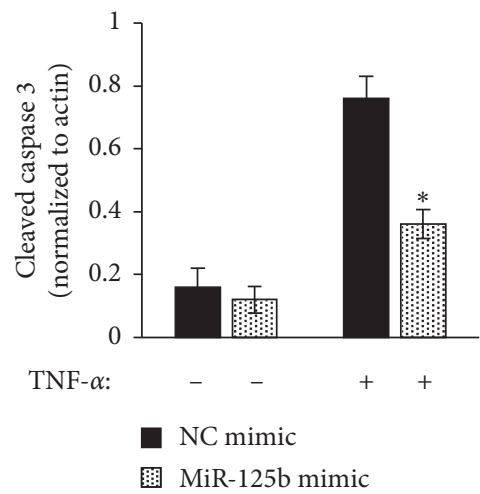

(d)

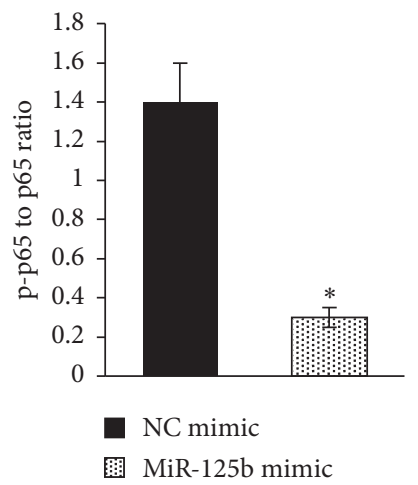

(f)

Figure 2: Upregulation of miR-125b in EPCs attenuated the expression of proinflammatory factors and ameliorated the TNF- $\alpha$-induced cell apoptosis. EPCs were treated with TNF- $\alpha(10 \mathrm{ng} / \mathrm{mL})$ for $1 \mathrm{~h}$ after transfection. The mRNA levels of proinflammatory factors (TNF- $\alpha$, IL-1 $\beta$, and IL-6) were measured by qRT-PCR (a). Cell apoptosis was determined by flow cytometry using annexin V staining (b). The activation of caspase 3 was analyzed by western blotting. Representative (c) and quantification (d) of caspase 3 level (normalized to $\beta$-actin). The activation of NF- $\kappa$ B was determined by the level of p-p65 in EPCs using western blotting. Representative (e) and quantification (f) of p-p65 level (normalized to $\mathrm{p} 65)\left({ }^{*} p<0.05\right.$ vs. NC mimic with TNF- $\alpha$ treatment, $\left.n=5\right)$.

\section{Results}

3.1. Overexpression of miRNA-125b in EPCs Ameliorates TNF$\alpha$ Induced Functional Defects in EPCs. The level of miR-125b in the EPC transfected with miR125b mimic is about 21folds increase compared with the negative control (NC) group (Figures 1(a) and 1(b)). Transfected EPCs were treated with or without $10 \mathrm{ng} / \mathrm{mL} \mathrm{TNF}-\alpha$ for $1 \mathrm{~h}$ and then tested for cell migration and adhesion in vitro. The NC group showed significant reduced cell migration (Figures $1(\mathrm{c})$ and $1(\mathrm{~d})$ ) and adhesion (Figures 1(e) and 1(f)) capacity after TNF- $\alpha$ treatment compared with the one without TNF- $\alpha$ treatment, while TNF- $\alpha$ treatment slightly reduced the cell migration and adhesion function in the miR-125b-overexpressed group in comparison with cells without TNF- $\alpha$ treatment. After TNF- $\alpha$ treatment, miR-125b-overexpressed EPCs 


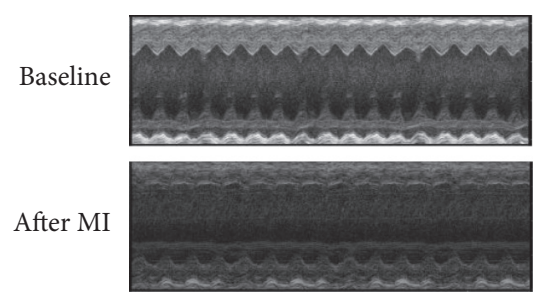

(a)

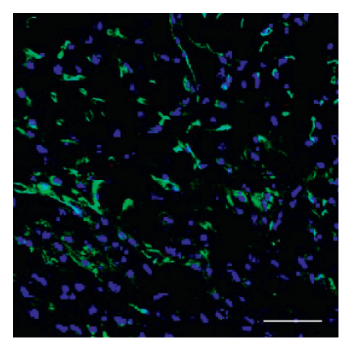

$\mathrm{NC}$

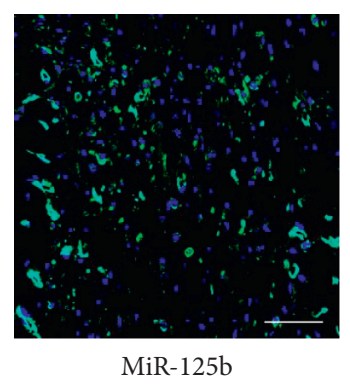

(c)

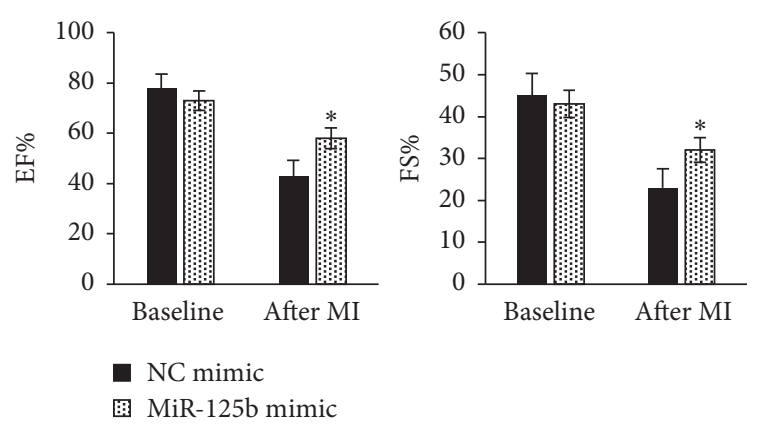

(b)

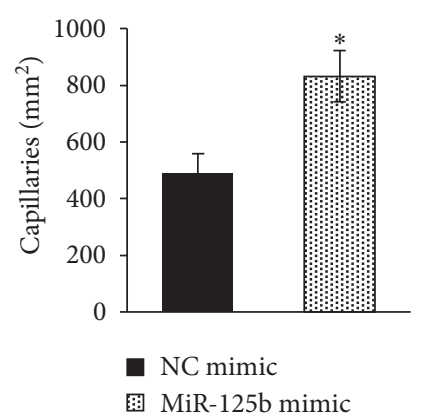

(d)

Figure 3: miR-125b overexpression enhanced the EPC-mediated neovascularization and cardiac function recovery in the ischemic hearts. The cardiac function was assessed by echocardiography at baseline and after MI (28 days). Representative (a) and quantification (b) of echocardiography analyses. EPC-mediated neovascularization in the ischemic hearts analyzed by CD31 staining (GFP) (c) and capillary density quantified (d) (scale bar $=100 \mu \mathrm{m},{ }^{*} p<0.05$ vs. NC mimic, $n=5$ ).

showed significantly better preserved migration and adhesion function than those of the NC group.

\subsection{Overexpression of miRNA-125b in EPCs Attenuates TNF-} $\alpha$ Induced Expression of Proinflammatory Factors and Cell Apoptosis. The mRNA level of the proinflammatory cytokines (TNF- $\alpha$, IL-1 $\beta$, and IL-6) in EPCs was measured by qRT-PCR after $1 \mathrm{~h}$ TNF- $\alpha$ treatment. We found that miR125b-overexpressed EPCs showed significantly lower TNF$\alpha$, IL- $1 \beta$, and IL- 6 mRNA expression levels than those of the NC group (Figure 2(a)). Moreover, the percentage of apoptotic cells (Annexin $V$ positive cells) is significantly lower in the miR-125b-overexpressed group compared with that of the NC group after TNF- $\alpha$ treatment (Figure 2(b)). In parallel with these flow cytometry results, our western blotting results showed markedly lower cleaved caspase 3 level in the overexpression group than in the NC group after TNF- $\alpha$ treatment (Figures 2(c) and 2(d)) which indicated reduced cell apoptosis.

3.3. Effects of miRNA-125b on EPC-Mediated Neovascularization in the Ischemic Hearts. To investigate the effect of miR-125b on the regulation of TNF- $\alpha$ induced NF$\kappa \mathrm{B}$ pathway activation, western blotting was performed to analyze the level of $\mathrm{p} 65$ phosphorylation ( $\mathrm{p}-\mathrm{p} 65)$. As shown in Figures 2(e) and 2(f), the level of p-p65 in miR-125boverexpressed EPCs was markedly lower than of the NC group after TNF- $\alpha$ treatment. To further investigate the role of miRNA-125b in EPC-mediated neovascularization, miR125b-overexpressed and NC control EPCs were transplanted by intramyocardial injections into the mice immediately after surgical-induction of MI, the cardiac function was evaluated by echocardiography, and the capillary density in the infarct border zone was assessed by immunohistology staining of CD31. The cardiac function of the mice transplanted with miR-125b-overexpressed EPCs was improved compared with that of the ones transplanted with $\mathrm{NC}$ control EPCs 28 days after MI (Figures 3(a) and 2(b)). The capillary density in the infarct border zone of the mice transplanted with miR-125b-overexpressed EPCs was about 2 -folds higher than the ones transplanted with $\mathrm{NC}$ control EPCs (Figures 3(c) and 2(d)). These data suggest miR-125b overexpression in EPC-enhanced EPC-mediated neovascularization and cardiac function recovery in the ischemic hearts.

\section{Discussion}

In this study, we have identified a novel role of miR-125b in the regulation of EPC functions. Upregulation of miR-125b in EPCs ameliorates the inflammatory and apoptotic responses of EPCs in the ischemic heart by inhibiting the activation of the TNF- $\alpha / \mathrm{NF}-\kappa \mathrm{B}$ pathway. Using the myocardial infarction model, we demonstrated that miR-125b overexpression enhanced the EPC-mediated neovascularization and cardiac function recovery in the ischemic hearts. To the best of our knowledge, this is the first 
study to address the role of miR-125b in modulating EPCmediated neovascularization in the ischemic hearts.

Although EPC-related cell therapy has been studied extensively, the majority of the cell-therapy trials achieve only modest efficacy [14-16]. The low survival rate of the transplanted EPCs in the ischemic hearts is one of the major obstacles to the success of this therapy $[17,18]$. After the myocardial infarction, a large number of inflammatory cells are recruited to the ischemic heart where a hostile, proinflammatory environment is created. Compelling evidence showed that the EPC level can be significantly affected by systemic inflammation [19]. It has been reported that lipopolysaccharide-induced systemic inflammatory reaction led to a decrease in the number of circulating EPCs [20]. Of note, patients with long-term inflammatory disease, like active ulcerative colitis, had significantly lower levels of circulating EPCs [21]. In this study, our data indicate that TNF- $\alpha$ treatment in EPCs markedly impaired its migration and adhesion function. More importantly, EPCs treated with TNF- $\alpha$ showed increased cell apoptosis, which might partly explain the low survival rate of transplanted EPCs in the ischemic hearts. Collectively, others and our study suggest that enhancing the survival of EPCs in the proinflammatory environment in the ischemic hearts is crucial for achieving satisfactory outcome of cell therapy.

miR-125 family is well known as one of the major regulators in the development of hematological malignancies and autoimmune diseases [22-24]. Recently, accumulating studies demonstrate that miR-125b negatively regulates the activation of the NF- $\kappa \mathrm{B}$ pathway by targeting TRAF6 [25]. More important, evidence showed that miR$125 \mathrm{~b}$-mediated repression of the NF- $\kappa \mathrm{B}$ pathway exerts a protective effect on ischemic hearts [13]. However, the role of miR125b in the regulation of EPC function is still unclear. Our study for the first time demonstrated that the overexpression of miR-125b led to resistance to TNF- $\alpha$-induced functional defects and cell apoptosis in EPCs and consequently enhanced the EPC-mediated neovascularization in the ischemic hearts. It is well recognized that the TNF- $\alpha / \mathrm{NF}-$ $\kappa \mathrm{B}$ pathway is central to most of the inflammatory processes and exerts negative regulatory effect on vascular repair [26]. In consistency with the reports from other cell types [27], our data showed that the overexpression of miR-125b blunted the TNF- $\alpha$ induced proinflammatory responses in EPCs and restored the functions of EPCs and, more importantly, protected against TNF- $\alpha$ induced apoptosis. Furthermore, mice transplanted with miR-125b-overexpressed EPCs showed enhanced neovascularization compared with that of the one with NC control EPCs. Taking together, our study for the first time unveils the protective effect of miR-125b on EPCs.

Although our data have demonstrated that upregulation of miR-125b blunted the TNF- $\alpha$-induced NF- $\kappa$ B pathway activation in EPCs, the detailed mechanisms underlying miR-125b-mediated negative regulation of NF- $\kappa$ B pathway in EPCs has not been revealed in this study. Reports from others have characterized TRAF6 as the target of miR-125b in the NF- $\kappa \mathrm{B}$ pathway activation $[13,25,27]$. However, whether miR-125b exerts its effect on TNF- $\alpha / N F-\kappa B$ pathway by targeting TRAF6 or other molecules in EPCs still requires further investigations to confirm.

In conclusion, our study suggests that miR-125b-mediated inhibition of the TNF- $\alpha / \mathrm{NF}-\kappa \mathrm{B}$ pathway is crucial for the protection of EPCs in the inflammatory environment and may be a novel therapeutic target for enhancing the effectiveness of cell therapy for ischemic heart disease.

\section{Data Availability}

The data used to support the findings of this study are included within the article.

\section{Conflicts of Interest}

The authors declare that they have no conflicts of interest.

\section{Authors' Contributions}

Ke Yang and Xing Liu contributed equally to this article.

\section{Acknowledgments}

This study was funded by the National Natural Science Foundation of China (81370214 to Chaoquan Peng).

\section{References}

[1] A. Samman Tahhan, M. Hammadah, M. Raad et al., "Progenitor cells and clinical outcomes in patients with acute coronary syndromes," Circulation Research, vol. 122, no. 11, pp. 1565-1575, 2018.

[2] M. Vasa, S. Fichtlscherer, A. Aicher et al., "Number and migratory activity of circulating endothelial progenitor cells inversely correlate with risk factors for coronary artery disease," Circulation Research, vol. 89, no. 1, pp. E1-E7, 2001.

[3] N. Werner, S. Kosiol, T. Schiegl et al., "Circulating endothelial progenitor cells and cardiovascular outcomes," New England Journal of Medicine, vol. 353, no. 10, pp. 999-1007, 2005.

[4] T. Eizawa, U. Ikeda, Y Murakami et al., "Decrease in circulating endothelial progenitor cells in patients with stable coronary artery disease," Heart, vol. 90, no. 6, pp. 685-686, 2004.

[5] N.-M. Heida, J.-P. Müller, I.-F. Cheng et al., "Effects of obesity and weight loss on the functional properties of early outgrowth endothelial progenitor cells," Journal of the American College of Cardiology, vol. 55, no. 4, pp. 357-367, 2010.

[6] H.-H. Xie, S. Zhou, D.-D. Chen, K. M. Channon, D.-F. Su, and A. F. Chen, "GTP cyclohydrolase I/BH4 pathway protects epcs via suppressing oxidative stress and thrombospondin-1 in salt-sensitive hypertension," Hypertension, vol. 56, no. 6, pp. 1137-1144, 2010.

[7] F. Mobarrez, L. Antoniewicz, J. A. Bosson, J. Kuhl, D. S. Pisetsky, and M. Lundback, "The effects of smoking on levels of endothelial progenitor cells and microparticles in the blood of healthy volunteers," PLoS One, vol. 9, no. 2, Article ID e90314, 2014.

[8] M. Golab-Janowska, E. Paczkowska, B. Machalinski et al., "Elevated inflammatory parameter levels negatively impact populations of circulating stem cells (CD133+), early endothelial progenitor cells (CD133+/VEGFR2+), and fibroblast growth factor in stroke patients," Current Neurovascular Research, vol. 16, no. 1, pp. 19-26, 2019. 
[9] P. Zhou, Y.-Z. Tan, H.-J. Wang, and G.-D. Wang, "Hypoxic preconditioning-induced autophagy enhances survival of engrafted endothelial progenitor cells in ischaemic limb," Journal of Cellular and Molecular Medicine, vol. 21, no. 10, pp. 2452-2464, 2017.

[10] A. Rodriguez, S. Griffiths-Jones, J. L. Ashurst, and A. Bradley, "Identification of mammalian microrna host genes and transcription units," Genome Research, vol. 14, no. 10a, pp. 1902-1910, 2004

[11] T. Chen, Z. Huang, L. Wang et al., "Microrna-125a-5p partly regulates the inflammatory response, lipid uptake, and ORP9 expression in oxLDL-stimulated monocyte/macrophages," Cardiovascular Research, vol. 83, no. 1, pp. 131-139, 2009.

[12] X. Zhao, Y. Tang, B. Qu et al., "MicroRNA-125a contributes to elevated inflammatory chemokine RANTES levels via targeting KLF13 in systemic lupus erythematosus," Arthritis \& Rheumatism, vol. 62, no. 11, pp. 3425-3435, 2010.

[13] X. Wang, T. Ha, J. Zou et al., "MicroRNA-125b protects against myocardial ischaemia/reperfusion injury via targeting p53-mediated apoptotic signalling and TRAF6," Cardiovascular Research, vol. 102, no. 3, pp. 385-395, 2014.

[14] M. Prasad, M. T. Corban, T. D. Henry, A. B. Dietz, L. O. Lerman, and A. Lerman, "Promise of autologous CD34+ stem/progenitor cell therapy for treatment of cardiovascular disease," Cardiovascular Research, vol. 116, no. 8, pp. 1424-1433, 2020.

[15] M. Teraa, R. W. Sprengers, R. E. G. Schutgens et al., "Effect of repetitive intra-arterial infusion of bone marrow mononuclear cells in patients with no-option limb ischemia," Circulation, vol. 131, no. 10, pp. 851-860, 2015.

[16] R. Flores-Ramírez, A. Uribe-Longoria, M. M. Rangel-Fuentes et al., "Intracoronary infusion of CD133+ endothelial progenitor cells improves heart function and quality of life in patients with chronic post-infarct heart insufficiency," Cardiovascular Revascularization Medicine, vol. 11, no. 2, pp. 72-78, 2010.

[17] D. C. S. Pedroso, A. Tellechea, L. Moura et al., "Improved survival, vascular differentiation and wound healing potential of stem cells co-cultured with endothelial cells," PLoS One, vol. 6, no. 1, Article ID e16114, 2011.

[18] H. K. Haider and M. Ashraf, "Strategies to promote donor cell survival: combining preconditioning approach with stem cell transplantation," Journal of Molecular and Cellular Cardiology, vol. 45, no. 4, pp. 554-566, 2008.

[19] S. Khodayari, H. Khodayari, A. Z. Amiri et al., "Inflammatory microenvironment of acute myocardial infarction prevents regeneration of heart with stem cells therapy," Cellular Physiology and Biochemistry, vol. 53, no. 5, pp. 887-909, 2019.

[20] F. B. Mayr, A. O. Spiel, J. M. Leitner, C. Firbas, W. Sieghart, and B. Jilma, "Effects of low dose endotoxemia on endothelial progenitor cells in humans," Atherosclerosis, vol. 195, no. 1, pp. e202-e206, 2007.

[21] J. Masuda, K. Mitsuyama, H. Yamasaki et al., "Depletion of endothelial progenitor cells in the peripheral blood of patients with ulcerative colitis," International Journal of Molecular Medicine, vol. 19, pp. 221-228, 2007.

[22] H. Zhang, X.-Q. Luo, D.-D. Feng et al., "Upregulation of microRNA-125b contributes to leukemogenesis and increases drug resistance in pediatric acute promyelocytic leukemia," Molecular Cancer, vol. 10, no. 1, p. 108, 2011.

[23] E. Tili, J.-J. Michaille, Z. Luo et al., "The down-regulation of mir-125b in chronic lymphocytic leukemias leads to metabolic adaptation of cells to a transformed state," Blood, vol. 120, no. 13, pp. 2631-2638, 2012.
[24] A. Ceribelli, B. Yao, P. R. Dominguez-Gutierrez, M. A. Nahid, M. Satoh, and E. K. Chan, "MicrorNAS in systemic rheumatic diseases," Arthritis Research \& Therapy, vol. 13, no. 4, p. 229, 2011.

[25] M.-S. Njock, H. S. Cheng, L. T. Dang et al., "Endothelial cells suppress monocyte activation through secretion of extracellular vesicles containing antiinflammatory micrornas," Blood, vol. 125, no. 20, pp. 320-3212, 2015.

[26] T. Inoue, K. Croce, T. Morooka, M. Sakuma, K. Node, and D. I. Simon, "Vascular inflammation and repair: iImplications for re-endothelialization, restenosis, and stent thrombosis," JACC: Cardiovascular Interventions, vol. 4, no. 10, pp. 10571066, 2011.

[27] C. Xie, L. Z. Zhang, Z. L Chen et al., "A hMTR4-PDIA3P1mir-125/124-TRAF6 regulatory axis and its function in $\mathrm{nf}$ kappa b signaling and chemoresistance," Hepatology, vol. 75, no. 5, pp. 1660-1677, 2019. 